\title{
Soluciones locales y flexibilidad en el conocimiento ecológico tradicional frente a procesos de cambio ambiental: estudios de caso en Patagonia
}

\author{
Marina Richeri²; María B. CARDOSO ${ }^{1} \&$ ANA H. LADIO ${ }^{1, \bowtie}$ \\ 1. INIBIOMA-CONICET-Universidad Nacional del Comahue, S.C. de Bariloche, Río Negro, Argentina. 2. CENPAT- \\ CONICET, Puerto Madryn, Chubut, Argentina
}

\begin{abstract}
RESUMEN. En este trabajo analizamos dos estudios de caso patagónicos que muestran la percepción de pobladores rurales acerca de los cambios ambientales y las soluciones locales, la flexibilidad de conocimientos y las prácticas en el uso de vegetales sustanciales para la vida cotidiana: las plantas leñateras y las medicinales. Se realizaron entrevistas semi-estructuradas y libres en comunidades pequeñas de ascendencia criolla y MapucheTehuelche en la meseta de la provincia de Chubut (Lagunita Salada y El Escorial) y de Río Negro (Pilquiniyeu del Limay). Se evaluó la percepción de los cambios ambientales en en relación al uso de plantas combustibles y medicinales. Se relevó la utilización actual de 45 especies medicinales ( $46 \%$ exóticas) y 27 especies combustibles (18\% exóticas). Los resultados sugieren que los pobladores, dentro del abanico de posibilidades, aplican sus saberes tradicionales con innovaciones tales como las forestaciones peridomésticas o los invernaderos, en donde se observa la utilización de especies útiles de origen exótico que generan indirectamente una menor presión sobre los recursos nativos. Estas soluciones locales indican procesos de resiliencia, desarrollados en un corto período de tiempo, articulando el conocimiento ecológico tradicional con las nuevas circunstancias socio-ambientales.
\end{abstract}

[Palabras clave: plantas leñateras, plantas medicinales, resiliencia socio-ambiental]

\begin{abstract}
Aвstract. Local solutions and flexibility in traditional ecological knowledge against environmental change processes: case studies in Patagonia: In this paper we analyzed two case studies of Patagonia showing perception of rural people about environmental changes and local solutions that reflect flexibility of traditional knowledge and practices regarding the use of plants that are substantial to their daily life, like firewood and medicinal plants. We studied, through randomly semi-structured and free interviews, the perception of environmental changes in the use of firewood and medicinal plants in descent Mapuche-Tehuelche and creole's small communities of the highlands of the province of Chubut (Lagunita Salada and El Escorial) and Río Negro (Pilquiniyeu of Limay). The current use of 45 medicinal species (46\% exotic) species and 27 firewood species ( $18 \%$ exotic) was recorded. Results show that people, as far as possible, apply their traditional knowledge and innovations such as peri-domestic afforestation, greenhouses and utilization of useful species of exotic origin that indirectly reduced pressure on native resources. These solutions indicate local resilience and self-processes that have been developed in a short period of time, articulating traditional ecological knowledge with new socio-environmental circumstances.
\end{abstract}

[Keywords: woody plants, medicinal plants, social and environmental resilience]

\section{INTRODUCCIÓN}

La mayoría de las comunidades rurales patagónicas dependen directa e indirectamente de los servicios ecosistémicos locales, por lo que deben ajustar prácticas y saberes en forma permanente ante los cambios. Estas prácticas ligadas al uso de la Naturaleza constituyen el conocimientoecológicotradicional("Traditional Ecological Knowledge, TEK") definido como un cuerpo de saberes y prácticas que es adquirido a lo largo de la historia, a través de la experiencia directa con el medio ambiente, que responde flexiblemente a cambios socio-ambientales y que se perpetúa por transmisión cultural (Berkes et al. 2000).
Estas actividades responden a necesidades vitales de subsistencia, como el manejo familiar del ganado ovino [que es la principal actividad productiva (Bandieri 2005)] y/o la recolección de plantas medicinales, que cumple un rol fundamental en su sistema tradicional de salud, además de responder a la escasa presencia de la medicina oficial en estos sitios alejados (Estomba et al. 2006; Molares \& Ladio 2010; Richeri et al. 2012). Por otra parte, son sustanciales el cultivo familiar a pequeña escala de frutas y hortalizas (Eyssartier et al. 2011a,b) y la recolección de leña como único medio para la calefacción y la cocción de alimentos (Cardoso et al. Nueva versión: 5 de julio; Aceptado: 19 de agosto. 
2012a,b). Según Berkes \& Davidson-Hunt (2010) y Toledo (2007), estos conocimientos locales permitirían afrontar las adversidades provocadas por disturbios o cambios de largo y corto plazo. Estos sistemas serían resilientes; ante determinados cambios del entorno contienen los componentes y los mecanismos necesarios para que los saberes y prácticas (e.g., sobre plantas) se reorganicen, se innoven, se renueven y se adapten para el bienestar de la comunidad (Berkes et al. 2000; Ladio 2011b).

Las poblaciones rurales originarias de la Patagonia han sufrido sucesivos cambios socio-ambientales, transformaciones culturales y ecológicas debido en gran parte a la influencia hegemónica de la sociedad de mercado, con lógicas del uso del ambiente que en la mayoría de los casos implicaron su sobreexplotación o transformación completa. Este nuevo orden económicoproductivo impuesto desde finales del siglo XIX se encuentra ligado a la producción extensiva de ganado ovino (Paruelo et al. 2006; Golluscio et al. 2010) o la forestación con especies exóticas. Al mismo tiempo, las poblaciones locales fueron diezmadas y sus territorios usurpados de manera que sus actividades productivas se restringen a la mera subsistencia. Además, el mayor acceso a la sociedad global y la mayor presencia de las instituciones en estos sitios aislados (Izquierdo et al. 2009; Eyssartier et al. 2011a), los procesos de des-agrarización (Eyssartier et 2011 a,b), migración (Gundermann et al. 2009), cambio de uso de la tierra y el creciente desarrollo de la megaminería de oro y plata (Gutiérrez 2006) son algunos otros procesos que vienen suscitando ajustes y cambios en la vida de esos pobladores.

Los cambios ambientales y socioculturales que se dan a lo largo del tiempo son percibidos muchas veces por los pobladores locales en términos de una comparación con la vida de sus ancestros en el lugar. Se ha postulado que dicha percepción sobre el entorno se articula con los conocimientos y prácticas locales (TEK), se ajustan a las nuevas condiciones y desencadenan procesos de resiliencia. En este sentido, un estudio realizado en Patagonia con comunidades rurales mapuches mostró que aquellos pobladores que conservan prácticas tradicionales (e.g, el piñoneo, la caza y el mantenimiento de la lengua Mapuzungun) poseen mayores conocimientos ambientales $\mathrm{y}$, en consecuencia, son más resilientes que aquellos que ya han dejado de reproducir sus tradiciones en la actualidad (Ladio 2011a).

Algunos autores (Soares Ferreira et al. 2011; Ladio 2011b; Cardoso et al. 2012b; Richeri et al. 2012) han propuesto que los recursos que ofrecen mayor versatilidad para una función y las prácticas que ofrecen mayor redundancia de recursos son las opciones principalmente elegidas por las comunidades, y que las mismas conllevan a una mayor flexibilidad y un uso más eficiente de los recursos naturales. En este trabajo analizamos y discutimos dos estudios de caso: el uso de plantas leñateras en la comunidad rural de Pilquiniyeu del Limay (Río Negro) y el uso de plantas medicinales en las comunidades rurales de la meseta chubutense de Lagunita Salada y El Escorial. En cada caso propusimos los siguientes objetivos: 1) estudiar la percepción ambiental de los cambios en cada sitio; 2) analizar la flexibilidad y el ajuste del TEK ante los cambios, focalizándonos en los componentes y los mecanismos que se manifiestan como soluciones locales. Nuestras principales preguntas fueron: ¿evidencian los pobladores cambios en la provisión de estos recursos? ¿cuáles son las soluciones locales, en términos de componentes y mecanismos, para mitigar dichos cambios? Nuestras hipótesis generales postulan que: las especies vegetales nativas y exóticas (los componentes del sistema) utilizadas en la actualidad por las comunidades han sido seleccionadas de manera tal que les permite a los pobladores mitigar la provisión de leña y/o los problemas de salud más frecuentes, brindando una relativa autosuficiencia. Por otra parte, existen diversos mecanismos que se articulan, los cuales propician el uso más eficiente y sostenible de los recursos silvestres.

\section{MÉTODOS}

\section{Sitios de estudio}

Ambos estudios se realizaron en poblaciones pequeñas de ascendencia Mapuche (Pilquiniyeu del Limay, Río Negro) y Mapuche-Tehuelche (Lagunita Salada y El Escorial, Chubut) que habitan áreas esteparias de la Patagonia, correspondientes a las regiones fitogeográficas del Monte y Patagónica (Cabrera 1976). Las dos poblaciones comparten la necesidad de sobrellevar rigurosas condiciones climáticas, así como también una elevada situación de aislamiento, debido al difícil acceso a las comunidades y las condiciones de falta de servicio público en general. En ambos poblados, la principal actividad económica es la cría de ganado ovino y 
caprino, la venta de artesanías y, en menor medida, el empleo en tareas de servicios públicos. En la mayor parte de los hogares visitados se practica el cultivo de la tierra con la presencia de huertos domiciliarios. En materia de salud pública, cuentan con un puesto de enfermería atendido por un agente sanitario local que brinda exclusivamente atención primaria y vacunación. Por un lado, Lagunita Salada y El Escorial $\left(42^{\circ} 31^{\prime} \mathrm{S}\right.$ y $69^{\circ} 24^{\prime} \mathrm{O}$, altitud 960 m.s.n.m.) se encuentran en el centro norte de la provincia del Chubut y están conformadas por 48 familias en total (INDEC 2001), con grandes lazos de parentesco, por lo que fueron tratadas como un solo estudio de caso. Lagunita Salada y la Aldea Escolar El Escorial están localizadas en un paisaje de sierras, valles y afloramientos rocosos, con una vegetación propia de la estepa arbustiva-herbácea (Beeskow et al. 1987). El clima es seco y frío (temperatura media anual $11.3^{\circ} \mathrm{C}$ y precipitación media anual $174 \mathrm{~mm}$ ). Por otra parte, Pilquiniyeu del Limay (898 m.s.n.m.) se encuentra localizada en la Región Sur de Río Negro $\left(41^{\circ} 02^{\prime} \mathrm{S}\right.$ y $70^{\circ} 51^{\prime}$ O) y está conformada por $\sim 55$ familias en un territorio declarado reserva indígena. Su relieve está formado por valles y mallines, como así también afloramientos rocosos. La vegetación se encuentra conformada principalmente por especies arbustivas formando un matorral (Cabrera 1976). Las especies nativas comúnmente encontradas son: Larrea nitida (jarilla), Colliguaja integerrima (coliguay), Schinus spp. (molles) y Lycium spp. (montenegro). El clima es seco y frío (temperatura media anual oscila entre 8 y $10^{\circ} \mathrm{C}$, y las precipitaciones entre 150 y $300 \mathrm{~mm} /$ año). La comunidad está conformada por un paraje en el cual viven 9 familias y un área dispersa donde se asientan $\sim 46$ hogares.

\section{Metodología etnobotánica y análisis de datos}

En primera instancia se obtuvieron los permisos de las autoridades locales y se obtuvo el consentimiento informado del entrevistado (CPI), de acuerdo a lo establecido en el Código de Ética Etnobiológica (ISE 2006). La aproximación fue cuali-cuantitativa, e incluyó el análisis de discursos y entrevistas con distinto grado de estructuración (Albuquerque et al. 2010). Se realizaron 24 entrevistas libres y semi-estructuradas ( $50 \%$ del total de viviendas) en Lagunita y El Escorial y 28 entrevistas en Pilquiniyeu del Limay (51\% de la población). En ambos casos se contó con la participación de un adulto voluntario en cada uno de los hogares visitados. También se recurrió a la utilización de herbarios y material fresco, además de realizar recorridos junto a informantes y observación participante (Albuquerque et al. 2010). La mayoría de las entrevistas fueron grabadas de acuerdo al consentimiento del informante. En todos los casos se desarrolló una lista de preguntas abiertas referentes a la percepción de los cambios de abundancia sobre los recursos de referencia (leñateras o medicinales, en el ambiente local, sus posibles causas así como también se indagó sobre los componentes del dominio (número de especies citadas por persona por medio de un enlistado libre) y los mecanismos (prácticas y/o soluciones locales para mantener su utilización, indagadas por medio de preguntas semi-estructuradas).

El material vegetal recolectado fue acondicionado para su posterior identificación e incorporación a la colección preservada del Jardín Botánico de la Patagonia Extraandina (CENPAT) y en el Laboratorio Ecotono (INIBIOMA). Los ejemplares fueron identificados y determinados siguiendo a Correa (1969, 1971, 1984, 1988a, 1988b, 1999) y Zuloaga \& Morrone (1999a,b). La nomenclatura científica fue actualizada consultando la base de datos del Catálogo de Plantas Vasculares del Conosur: http://www2.ddarwin.edu.ar / Proyectos/FloraArgentina/FA.asp.

La percepción del cambio en los últimos 60 años en las comunidades se basó en un análisis cualitativo que incluyó la interpretación de narrativas locales sobre la riqueza y abundancia de los recursos vegetales en cuestión. La percepción de cambios en los recursos vegetales útiles se analizó tomando como referencia las entrevistas y los relatos de personas que residieron en la región por más de 60 años. Siguiendo a Suárez (2009), este tipo de indagaciones permite al informante reelaborar y recontextualizar los significados a medida que formulan sus relatos, en este caso sobre la disponibilidad de los recursos útiles y las causas de su posible disminución.

La riqueza total de especies vegetales citadas por las comunidades se estimó teniendo en cuenta el número de plantas que fueron citadas por la totalidad de los entrevistados en cada comunidad (Ladio \& Lozada 2003, 2004a). Las especies utilizadas en cada comunidad se clasificaron según su origen biogeográfico en nativas y exóticas siguiendo a Zuloaga \& Morrone (1999a, b). Para determinar la importancia de cada especie dentro de las comunidades rurales, se estimaron dos índices. Por un lado se calculó el Consenso de Uso (CU), para cada una de las especies citadas, tomando el número de especies mencionadas por persona sobre el total de informantes, siendo este valor indicativo de la intensidad o frecuencia de uso de las especies para cada una de las comunidades (Ladio \& Lozada 2008).

Por otra parte, se aplicó el Índice de Significancia Cultural (ISC) propuesto por Silva et al. (2006): ISC $=\Sigma(\mathrm{i} * \mathrm{e} * \mathrm{c}) * \mathrm{CF}$, donde $\mathrm{i}$ representa el grado de manejo de la especie $(2=$ especie cultivada y $1=$ especie recolectada); e indica la preferencia de uso (2=uso preferencial; $1=$ uso alternativo; c refleja la frecuencia de uso ( $2=$ plantas efectivamente conocidas y usadas; 1 =plantas raramente citadas), y por último $\mathrm{CF}$ es el factor de corrección asociado al consenso de uso de la planta (CU). La versatilidad de cada especie medicinal se expresó a partir de la suma total de usos (i.e., si una especie se usa como digestiva pero también como antifebril, 
su versatilidad será igual a 2), al igual que para las especies leñateras (i.e.. una especie puede ser utilizada para leña, forraje o mueblería, y dentro de la categoría de uso leña también puede ser utilizada para encender el fuego, como leña dura y gruesa para mantener el fuego o como leña para cocinar ladrillos). En este caso, cada uso perteneciente a las categorías de uso principales o dentro de la subcategoría leñateras, se consideró como un uso diferente, sumando cada uno por separado al valor de la versatilidad total de cada especie. El análisis de la información cuantitativa se llevó a cabo utilizando pruebas no paramétricas (Höft et al. 1999). Se utilizó la prueba binomial para testear diferencias en el uso de plantas nativas y exóticas dentro de cada una de las comunidades; se aplicó el estadístico U de Mann Whitney para detectar diferencias en la variable consenso de uso (CU) entre comunidades y además, se realizaron correlaciones de Spearman con el fin de explorar si el consenso de uso está asociado con la versatilidad de cada una de las especies (Conover 1980).

\section{Resultados y Discusión}

\section{Percepción ambiental del cambio}

En ambos estudios se encontró que la riqueza y abundancia relativa de especies medicinales silvestres en las comunidades de Chubut y leñateras en la población de Río Negro disminuyeron en comparación con décadas anteriores según la percepción expresada en los relatos del $100 \%$ de los entrevistados.

En el caso de Lagunita Salada y El Escorial, las entrevistas revelan que los pobladores rurales perciben una disminución en las plantas medicinales-tomandocomoparámetro60años atrás, momento de la apertura de la escuela, y que este cambio no se distribuye por igual en el paisaje de mesetas. En general, los pobladores diferencian las zonas altas (los "cerros") y las bajas (el "campo abierto"). En los primeros, según la percepción local, ha disminuido la abundancia de plantas. Por el contrario, en el "campo abierto" los pobladores reconocen una mayor disminución en la riqueza de ejemplares medicinales. Los pobladores de este sitio, argumentan las disminuciones apelando a cambios en los ciclos naturales que sufre la región y la extensa sequía, la mayor cantidad de gente en los poblados y/o la actividad de exploración minera de la zona. Algunos informantes consideran la totalidad de factores como si estuvieran actuando de manera simultánea. En la mayor parte de los relatos, los informantes expresan que a la gente del pueblo no le queda otra opción que salir a buscar plantas en parajes más alejados por lo que cada vez tardan mayor tiempo en encontrar el remedio buscado. Además la totalidad de los entrevistados perciben cambios climáticos tendientes a recrudecer la sequía propia del lugar como la disminución lluvias y nieve que hacen que las vertientes se sequen y desaparezcan junto con las plantas medicinales que allí se desarrollaban. En general, el análisis de entrevistas pertenecientes a los pobladores más antiguos nos muestra que las distancias para recolectar las plantas medicinales son cada vez más largas y que es motivo de preocupación para toda la comunidad. Las referencias a la sequía y a la falta de agua están cargadas de tristeza y desazón, y algunos hasta lo ven como un castigo. También se evidencia cierta nostalgia por el pasado, cuando la búsqueda de remedios no representaba un problema como lo es hoy. Además. se percibe que los cambios producidos por la actividad minera de la zona generan mucha incertidumbre en la gente.

En el caso de los pobladores de Pilquiniyeu del Limay, los informantes coincidieron con las razones vinculadas a la disminución de leña en el lugar en términos tanto de riqueza como de abundancia relativa. Estos informantes citan a la sequía y al uso constante en el lugar como primeras razones, y exceptúan a la intromisión minera, que no es todavía una actividad prevalente en la zona. Del mismo modo que el caso anteriormente descripto, la búsqueda de la leña implica viajes cada vez más largos, dado que su obtención es vital para el desarrollo de su vida cotidiana y subsistencia. El análisis de las entrevistas también evidencia desazón acerca de la falta de agua, y nostalgia por un pasado en el que las condiciones para la búsqueda de leña eran mejores. Se denota cómo el traslado de la comunidad a otra localización geográfica es recordado con pesar, y cómo las condiciones socioeconómicas actuales son vividas con malestar e impotencia. Por otra parte, también se hace referencia a una sobreexplotación pasada de las plantas leñateras en la zona, hecho que ha acarreado una disminución actual del recurso. Otros autores han descripto procesos similares en otras regiones áridas (Romo et al. 1999; Lucena et al. 2007). De esta forma, las opiniones y los sentimientos expresados en los discursos, quedan lejos de ser aleatorios y revelan que para ambos grupos sociales los cambios ambientales son elementos substanciales de su discurso y, por 
ende, conllevan una valoración que implica actitudes y acciones consecuentes.

\section{Soluciones locales y flexibilidad del conocimiento ecológico tradicional}

Frente a los cambios en la disponibilidad de plantas útiles en ambas comunidades los pobladores de ambas sitios de estudio generan soluciones locales, que fuera de establecer si son óptimas o no, representan formas de ajuste a las condiciones actuales. Estas soluciones están conformadas por ciertos componentes biológicos como las especies de plantas, y ciertos mecanismos que describiremos a continuación.

Las soluciones locales se basan sobre un conjunto de especies útiles para los pobladores (componentes), las cuales permiten suplir sus necesidades en cuanto a las categorías de uso estudiadas. En el estudio de caso de Lagunita Salada y Escorial se encontró que se utiliza una riqueza total de sólo 45 especies medicinales. No se registraron diferencias significativas en relación al uso de plantas nativas y exóticas (prueba binomial; $P=0.3$ ); las nativas están representadas por 26 especies $(58 \%)$ y las exóticas por 19 especies (42\%). Entre las exóticas, $76 \%$ son cultivadas en huertas e invernaderos, y sólo $26 \%$ son silvestres. La riqueza de especies registrada aquí resulta baja en relación a lo descripto por otros autores en Patagonia: Conticello et al. (1997) registraron 96 especies, Estomba et al. (2006) describieron 89 y Molares \& Ladio (2008) citan 131 especies medicinales. Estos resultados sugerirían que la aridez del sistema ocasiona una menor oferta ambiental de especies medicinales en la meseta central chubutense, tanto nativas como exóticas.

En relación a la aplicación medicinal específico de las plantas citadas, encontramos que $36 \%$ de las especies son utilizadas para combatir dolencias gastrointestinales, seguidas por las respiratorias $(14 \%)$, y en menor medida para combatir afecciones menores y/o poco frecuentes como las inflamatorias, dermatológicas, o la fiebre, entre otras. Estas dolencias también fueron encontradas en otras áreas rurales patagónicas (Estomba et al. 2006; Molares \& Ladio 2010), hecho que demuestra que los componentes adoptados son sustanciales para paliar las dolencias más prevalentes, y por ende, no son elegidos al azar.
Las especies medicinales con mayor consenso de uso entre los pobladores de Lagunita Salada y El Escorial son tanto nativas como exóticas (U de Mann Whitney=0.66; P<0.05). Este resultado abona la idea de que los pobladores han construido una herbolaria compleja basada sobre la interacción entre conocimientos nuevos y tradicionales. El cultivo de plantas medicinales (e.g., ver en Tabla 1, Mentha spicata "menta pastilla", Artemisia absinthium "ajenco", Matricaria recutita "manzanilla") permite el crecimiento y la obtención de especies importantes para determinadas dolencias, al mismo tiempo que reduce el esfuerzo de búsqueda y presión de uso en relación a las plantas silvestres. Sin embargo, estas últimas son muy importantes en términos culturales, ya que no sólo tratan varias dolencias, sino que también son utilizadas frecuentemente (i.e., ver en Tabla 1 Clinopodium darwinii "té pampa", Acantholippia seriphioides "tomillo silvestre", Nardophyllum bryoides "sietecamisas" Chuquiraga avellanedae "quilimbay", y Valeriana spp. "ñancolahuen").

En Pilquiniyeu del Limay se encontró una riqueza total de 27 plantas leñateras, de las cuales 22 son especies de arbustos nativos de la región (82\%) y 5 especies son árboles de origen exótico $(18 \%)$ (Test Binomial, $P=0.002)$. Todas las plantas exóticas son árboles cultivados de crecimiento rápido (Cardoso \& Ladio 2011) con posibilidades de adaptarse a las condiciones de sequía, el viento y / o el forrajeo de los animales. Las especies que presentaron el mayor consenso de uso son las nativas, tales como los Schinus spp. (molles), Monttea aphylla (yaque), Larrea nitida (jarilla), Lycium spp. (montenegro), Senecio subulatus (romerillo) y Prosopis denudans (alpataco) (Tabla 1). En el caso de las especies cultivadas introducidas, el consenso de uso se encuentra muy por debajo, comparándolo con el consenso para las especies nativas (Tabla 1). Este hecho se debe a que la flora nativa es preferida por los pobladores por la calidad de su madera que presenta mayor dureza, y por lo tanto mayor duración en la combustión (Cardoso et al. 2012a). Además, existe en esta comunidad una mayor disponibilidad del recurso nativo, ya que las especies cultivadas con maderas más blandas y de rápido crecimiento se encuentran sólo en el sector peridoméstico de los asentamientos (Cardoso \& Ladio 2011). Este hecho demuestra el valor del conocimiento ecológico tradicional en la elección diaria de los recursos, al mismo tiempo enriquecido por 
Tabla 1. Lista de especies vegetales útiles de cada sitio de estudio, ordenada según su Consenso de Uso (CU).

Table 1. List of useful plant species of each site, ordered by Consensus of Use (CU).

\begin{tabular}{|c|c|c|c|c|c|}
\hline $\begin{array}{l}\text { Especies medicinales } \\
\text { (Lagunita Salada y El Escorial) }\end{array}$ & Origen & $\mathrm{CU}$ & $\begin{array}{l}\text { Especies leñateras } \\
\text { (Pilquiniyeu) }\end{array}$ & Origen & $\mathrm{CU}$ \\
\hline Mentha spicata $x$ aquatica & Cultivada & 43 & Schinus johnstonii & Silvestre & 82 \\
\hline Acantholippia seriphioides & Silvestre & 39 & Monttea aphylla & Silvestre & 64 \\
\hline Satureja darwinii & Silvestre & 39 & Schinus marchandii & Silvestre & 64 \\
\hline Artemisia absinthium & Cultivada & 35 & Larrea nitida & Silvestre & 50 \\
\hline Nardophyllum obtusifolium & Silvestre & 35 & Lycium ameghinoi & Silvestre & 50 \\
\hline Valeriana carnosa & Silvestre & 30 & Senecio subulatus & Silvestre & 50 \\
\hline Chuquiraga avellanedae & Silvestre & 30 & Prosopis denudans & Silvestre & 36 \\
\hline Matricaria recutita & Cultivada & 30 & Colliguaja integerrina & Silvestre & 32 \\
\hline Larrea nitida & Silvestre & 26 & Lycium chilense & Silvestre & 29 \\
\hline Fabiana imbrincata & Silvestre & 26 & Ochetophila trinervis & Silvestre & 29 \\
\hline Erodium cicutarium & Silvestre & 26 & Salix viminalis & Cultivada & 25 \\
\hline Adesmia boronioides & Silvestre & 26 & Corynabutilon bicolor & Silvestre & 18 \\
\hline Grindelia chilense & Silvestre & 26 & Berberis microphylla & Silvestre & 11 \\
\hline Sedum telephium & Cultivada & 26 & Chuquiraga erinaceae & Silvestre & 7 \\
\hline Marrubium vulgare & Silvestre & 22 & Nassauvia axillaris & Silvestre & 7 \\
\hline Melissa officinalis & Cultivada & 22 & Populus nigra & Cultivada & 7 \\
\hline Ephedra ochreata & Silvestre & 22 & Senecio filaginoides & Silvestre & 4 \\
\hline Dysphania multifida & Silvestre & 22 & Adesmia volckmanni & Silvestre & 4 \\
\hline Tanacetun vulgare & Cultivada & 22 & Atriplex lampa & Silvestre & 4 \\
\hline Usnea sp. & Silvestre & 17 & Lycium gilliesanum & Silvestre & 4 \\
\hline Ruta graveolens & Cultivada & 17 & Neosparton aphyllum & Silvestre & 4 \\
\hline Retanilla patagonica & Silvestre & 17 & Satureja darwinii & Silvestre & 4 \\
\hline Plantago lanceolata & Silvestre & 17 & Mulinum spinosum & Silvestre & 4 \\
\hline Buddleja globosa & Cultivada & 17 & Stillingia patagonica & Silvestre & 4 \\
\hline Senecio filaginoides & Silvestre & 17 & Populus $x$ canadensis & Cultivada & 4 \\
\hline Schinus johnstonii & Silvestre & 17 & Ulmus minor & Cultivada & 4 \\
\hline Acaena sp. & Silvestre & 13 & Populus alba & Cultivada & 4 \\
\hline Tetraglochin alatum & Silvestre & 13 & & & \\
\hline Tanacetun balsamita & Cultivada & 13 & & & \\
\hline Larrea ameguinoi & Silvestre & 9 & & & \\
\hline Polygonum sanguinaria & Silvestre & 9 & & & \\
\hline Peumus boldus & Cultivada & 9 & & & \\
\hline Prosopidastrum globosum & Silvestre & 9 & & & \\
\hline Atriplex lampa & Silvestre & 9 & & & \\
\hline Solanum tuberosum & Cultivada & 4 & & & \\
\hline Acaena sp. & Silvestre & 4 & & & \\
\hline Centaurium cachanlahuen & Silvestre & 4 & & & \\
\hline Conium maculatum & Silvestre & 4 & & & \\
\hline Dichondra sp. & Silvestre & 4 & & & \\
\hline Dysphania ambrosioides & Silvestre & 4 & & & \\
\hline Sambucus nigra & Cultivada & 4 & & & \\
\hline Artemisia abrotarum & Cultivada & 4 & & & \\
\hline Chrisantemun sp. & Cultivada & 4 & & & \\
\hline Cichorium intybus & Silvestre & 4 & & & \\
\hline Mulinum spinosum & Silvestre & 4 & & & \\
\hline
\end{tabular}


nuevos componentes, en este caso las especies utilizadas en la forestación peridoméstica.

El análisis de las entrevistas refleja la existencia de mecanismos comunes en ambas prácticas, los cuales, de manera conjunta, permitirían hacer un uso eficiente y flexible de los componentes elegidos. Estos mecanismos actúan de manera directa sobre los recursos vegetales y se ponen en acción junto con otros mecanismos socioculturales (e.g., la transmisión social de la información), que mantienen vivos los saberes acumulados en la experiencia con el ambiente (Berkes \& Davidson-Hunt 2006). Los mecanismos encontrados en este trabajo y que también fueron citados en la literatura pero no de manera integral son: 1) la versatilidad, como atributo selectivo sobre el "pool" de plantas utilizadas [i.e., las especies más usadas son las que permiten suplir distintos requerimientos en relación al uso, y por lo tanto las especies comúnmente utilizadas o con mayor consenso son las multifuncionales (Berkes 1999; Ladio \& Lozada 2008; Richeri et al. 2010)]; 2) la redundancia [i.e, la existencia de numerosos recursos que sirven para la misma función y que pueden ser utilizados de manera alternativa (Albuquerque \& Oliveira 2007; Ladio 2011a; Soares Ferreira et al. 2011)]; 3) la complementariedad de actividades [i.e., la actividad de recolección se asocia con la de cultivo o forestación u otras, y de esta manera se incrementan las posibilidades de obtener una riqueza mayor de componentes (Ladio \& Lozada 2004b; Eyssartier et al. 2009; Ladio 2011b)]; 4) los viajes multipropósito de búsqueda de los recursos [i.e., implican la minimización de costos energéticos y tiempos de viaje (Ladio \& Lozada 2004b)]; 5) la hibridación con saberes nuevos [i.e., el proceso que permite que se asimilen

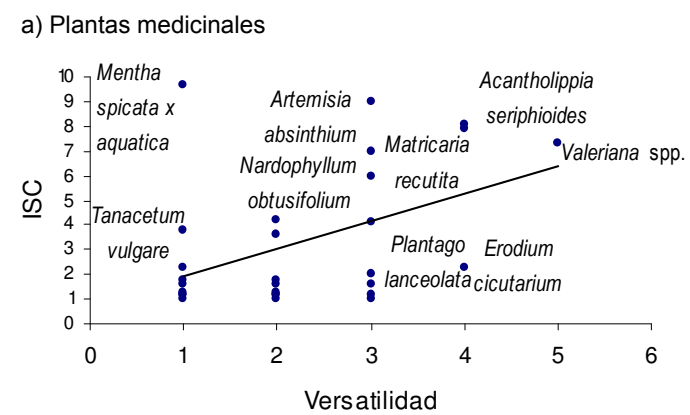

innovaciones y/o conocimientos nuevos, en general provenientes de los ámbitos científicos/tecnológicos al pool de saberes tradicionales (Ladio \& Molares 2010)], y 6) la presencia de prácticas de manejo que propician la perpetuación del recurso, y que implican valores y normas sociales que favorecen la sustentabilidad y el mantenimiento de la biodiversidad (González-Insuasti \& Caballero 2007; Blancas et al. 2010).

Los mecanismos comunes presentan particularidades para cada una de las prácticas de subsistencia analizadas que se describen a continuación. En Lagunita Salada y El Escorial: 1) el 100\% de personas usan plantas medicinales para solucionar sus problemas de salud más comunes. Este hecho denota que sus habitantes son, en términos de la atención primaria de la salud, autosuficientes en la medida que pueden proveerse de los recursos necesarios para tal fin. Además es significativo que, aunque su herbolaria se compone de pocas especies (45 especies), las plantas con mayor importancia cultural, es decir mayor ISC, son las más versátiles como es el caso de "ñancolahuen", "tomillo silvestre" y "ajenjo" (Figura 1a, Correlación de Spearman $\rho=0.46$, $\mathrm{n}=45, P<0.05)$, lo que muestra un ajuste posible de la herbolaria a las condiciones ambientales limitantes actuales. 2) Por otra parte, los remedios vegetales son redundantes para cada categoría de uso, es decir que existen varias especies que tratan la misma dolencia (i.e., las afecciones hepáticas pueden ser tratadas por Valeriana spp. "ñancolahuen", por Dysphania ambrosioides y D. multifida "paicos" y por Artemisia absinthium "ajenco". 3) La complementación de actividades es otro rasgo característico, $70 \%$ de las citas de plantas se obtienen por recolección, mientras que 30\% por cultivo, lo cual muestra simultáneamente

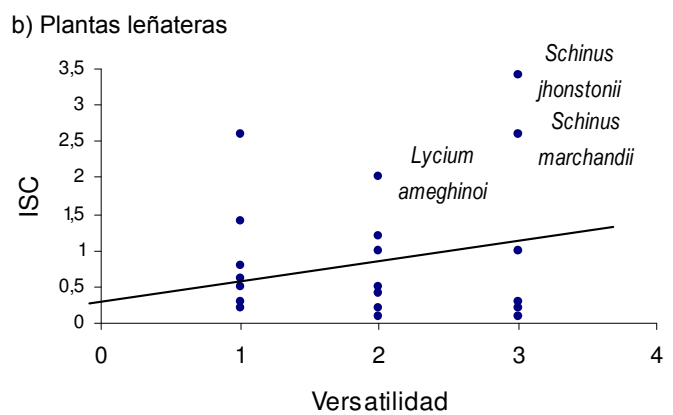

Figura 1. Asociación de Indice de Importancia Cultural (ISC) con la versatilidad de las especies citadas por los pobladores para: a) uso medicinal (Lagunita Salada y El Escorial, Chubut) y b) leña (Pilquiniyeu del Limay, Río Negro).

Figure 1. Association between Cultural Importance Index (ISC) and the versatility of the plant species named by the settlers for: a) medicinal use (Lagunita Salada) and b) fuel (Pilquiniyeu). 
una gran dependencia del ambiente natural, pero a su vez, la articulación con otras prácticas de manejo como la horticultura. El 63\% de los entrevistados incrementa la riqueza de plantas medicinales con huertas e invernaderos, lo cual aumenta el número de especies de 4 a 7 en promedio por familia. 4) El $21 \%$ de los informantes realizan viajes multipropósito de recolección, de manera que los esfuerzos energéticos se reparten con otras actividades (cría de ganado, búsqueda de leña, viajes por salud o visitas), de modo que sigue siendo posible la recolección de plantas lejanas a los hogares. 5) La herbolaria evidencia procesos de hibridización al incorporar especies introducidas al conjunto de recursos curativos (i.e., Mentha spp. "mentas", Matricaria recutita "manzanilla", Peumus boldus "boldo", entre otras). Los pobladores que provienen de otros sitios llevan consigo saberes y prácticas vinculados a las plantas (y en algunos casos incluso trasladan plantas de interés para cultivar y cosechar en el nuevo hogar) que luego son transmitidos e incorporados al resto de la población. Además, luego de analizar las entrevistas, observamos que podrían existir innovaciones en el uso de las especies introducidas mediante la exploración del entorno natural y la experimentación directa con las plantas poco conocidas como es el caso de la incorporación a la herbolaria local de Dysphania multifida "paico" y Acaena spp. "sillolahuen". Además, el uso de invernaderos constituye una de las innovaciones traídas por los extensionistas más aceptadas en la región que provee de nuevos recursos y/o promueve la extensión del tiempo de cosecha (Eyssartier et al. 2011a, b). 6) Por último, se despliegan prácticas sustentables de manejo de distinta índole; por ejemplo, el 33\% de los pobladores aprovecha las plantas invasoras dispersadas por el ganado (e.g., Erodium cicutarium, Marrubium vulgare, Clinopodium ambrosioides, entre otras), lo que demuestra una gran sintonía con los cambios del ambiente y un aprovechamiento diversificado. El 71\% de los entrevistados realiza el secado y almacenamiento de las especies que representan un mayor esfuerzo de búsqueda y obtención (en el caso de Adesmia boronioides "paramela", Valeriana spp. "ñancolahuen" y Fabiana imbricata "palopiche"), lo cual asegura su aprovisionamiento por mayor tiempo y un uso más eficiente del recurso medicinal. Por último, todos los entrevistados resaltaron que sólo hay que recolectar lo que se necesita para no sobreexplotar las plantas medicinales.
Por otra parte, los datos relevados en Pilquiniyeu del Limay muestran que: 1) existe una tendencia que refleja que las especies con mayor importancia cultural son las más versátiles, es decir que se emplean para distintas etapas del proceso de combustión y/o tienen otros usos asociados, sin embargo la misma no es significativa (Figura $1 b$, Correlación de Spearman $\rho=0.15, \mathrm{n}=27$, $P>0.05$ ). Es el caso de los "molles" que además de poseer una de las mejores maderas para leña, son utilizados para postes en los corrales o en los cercos, para medicina y para forraje, evidenciando su elevada importancia cultural. 2) Los recursos leñateros están representados por una riqueza total de 27 especies; de esta manera, el impacto de extracción se encuentra distribuido (22 especies silvestres nativas y en 5 especies cultivadas). Las distintas opciones de recolección, entonces, pueden reemplazarse unas con otras en función de su disponibilidad. 3) La complementación de actividades puede observarse en la realización de forestaciones peridomésticas con especies de árboles exóticos con diferentes fines (e.g., la obtención de leña). En esta comunidad, las forestaciones peridomésticas se componen aproximadamente de 9 especies, de las cuales 5 son aprovechadas para el uso leñero, como Populus spp., Salix spp. y Ulmus sp (Tabla 1). 4) El 79\% de la población entrevistada aprovecha otros viajes para la recolección de leña. La actividad vinculada más estrechamente a la recolección de leña es la crianza y cuidado diario del ganado, lo cual minimiza el costo energético y el tiempo de búsqueda. Asimismo, los viajes de visita entre vecinos también han sido citados como momentos donde también se aprovecha para recolectar este recurso. 5) La forestación es una práctica común en la región y es estimulada por la presencia de agentes de promoción rural, lo cual denota el grado de adaptación de esta innovación desde hace tiempo. 6) Se pusieron en evidencia distintas prácticas de manejo. El $64 \%$ de la población recolecta y utiliza leña caída o seca como único combustible. El 40\% complementa la escasez del recurso leñero con el uso de estiércol de vaca y caballo, y el 39\% queman en la estufa la leña seca junto con la leña verde. Esta mezcla resulta provechosa que que mantiene el poder de combustión y hace más eficiente el uso del recurso vegetal. También se evidenciaron normas sociales (ver Fragmento 3) a través de las cuales los pobladores establecen regulaciones en la extracción del recurso vegetal leñatero. Por 
ejemplo, como parte de las soluciones locales en esta comunidad, los pobladores han decidido desde el año 2009 no vender leña del monte nativo a terceros, excepto entre vecinos, como medida de cuidado de la riqueza local o de manejo relativamente sustentable.

\section{CONCLUSIÓN}

Los pobladores rurales del interior patagónico dan cuenta de los cambios registrados en relación a las condiciones climáticas, ecológicas y sociales que los atraviesan. Los variados componentes y mecanismos expuestos en este trabajo como soluciones locales reflejan, por un lado, el abanico de recursos útiles de la región aprovechables como medicina y leña. Y por otro lado, el grado de flexibilidad del conocimiento tradicional en la incorporación de nuevos saberes. Estos conocimientos aportan riqueza, ayudan a afrontar las nuevas condiciones del entorno, y permiten el desarrollo de patrones de sustentabilidad y el manejo local de los recursos. Por ejemplo, el cultivo de plantas exóticas (en huertas o forestaciones) para distintos fines genera indirectamente una presión menor sobre los recursos nativos. Estas soluciones locales podrían estar indicando procesos de resiliencia ecológico-social. En ciertos casos, estos procesos han sido desencadenados en períodos cortos de tiempo. Sin embargo, los relatos de las personas muestran que estas soluciones locales son estrategias que no alcanzan a paliar los problemas que los aquejan y representan en la mayoría de los casos ejemplos de supervivencia a condiciones muy duras. Entonces, si bien estas soluciones encontradas corresponden a prácticas de manejo adaptativo dado que les permite responder a los cambios sin perder opciones para el futuro, es necesario prestar una atención mayor a los procesos actuales, de modo de encontrar soluciones superadoras que mejoren la calidad de vida de los habitantes. Esto sólo es posible si las instituciones y organizaciones intervinientes empiezan a considerar la conservación desde una mirada no centrada sólo en los recursos biológicos, sino centrada en un sistema acoplado e indivisible ecológico-social.

Agradecimientos: Agradecemos especialmente a las familias de Pilquiniyeu del Limay, Lagunita Salada y El Escorial por su hospitalidad y amabilidad en compartir sus saberes. Además agradecemos a CONICET y a ANPCyT. Este trabajo ha sido financiado por los proyectos PICT 0702289 y PIP 0337 cuyo titular es la Dra. A. Ladio.

\section{BiBLIOGRAFÍA}

Albuquerque, UP \& RF De Oliveira. 2007. Is the use-impact on native Caatinga species in Brazil reduced by the high species richness of medicinal plants? J Ethnopharmacol, 113:156-170.

Albuquerque, UP; RF Paiva de Lucena \& LVF CRuz DA CunHa. 2010. Métodos e Técnicas na Pesquisa Etnobiológica y Etnoecológica. Ed. NUPPEA, Recife, Brazil.

BANDIERI, S. 2005. Historia de la Patagonia. Sudamericana S.A. Buenos Aires. Argentina.

Beeskow, AM; H Del Valle \& CM Rostagno. 1987. Los Sistemas Fisiográficos de la Región Árida y Semiárida de la Provincia del Chubut. Ed. SECYT. Puerto Madryn, Argentina.

BerKes, F. 1999. Sacred Ecology. Traditional ecological knowledge and resource management. Ed. Taylor \& Francis. Philadelphia y Londres.

Berkes, F \& IJ Davidson-Hunt. 2006. Biodiversity, traditional management systems, and cultural landscapes: examples from the boreal forest of Canada. Int. Soc. Sci. J., 87:35-47.

BERKES, F \& IJ DAVIDSON-Hunt. 2010. Innovating through commons use: community-based enterprises. Int. J. Commons, 4:1-7.

Berkes, F; J Colding \& C Folke. 2000. Rediscovery of traditional ecological knowledge as adaptive management. Ecol. Appl., 10:1251-1262.

Blancas, J; A Casas; S Rangel-Landa; A Moreno-Calles; I TorRES; ET AL. 2010. Plant Management in the TehuacánCuicatlán Valley, Mexico. Econ. Bot., 64:287-302.

Cabrera, AL. 1976. Regiones Fitogeográficas Argentinas. Acme S.A.C.I. Buenos Aires. Argentina.

CARDOSO, MB \& AH LAdIO. 2011. Forestación peridoméstica en Patagonia y conocimiento ecológico tradicional: un estudio de caso. Sitientibus série Ciências Biológicas, 11: 321-327.

Cardoso, MB; AH Ladio \& M Lozada. 2012a. The use of firewood in a Mapuche community in a semi-arid region of Patagonia. Biomass Bioenergy, 46:155-164.

Cardoso, MB; AH Ladio \& M Lozada. 2012b. Fuelwood consumption patterns and resilience in two rural communities of the Patagonian northwest steppe, Argentina. J. Arid Environ. En prensa.

CONOver, WJ. 1980. Practical Nonparametric Statistics. Ed. Wiley \& Son. New York.

Conticello, L; R Gandullo; A Bustamante \& C Tartaglia. 1997. El uso de las plantas medicinales por la comunidad mapuche de San Martín de los Andes, Prov. de Neuquén (Argentina). Parodiana 10:165-180.

Correa, MN. 1969-1999. Flora Patagónica. República Argentina. Ed. Colec. Cient. INTA.

Estomba, D; AH Ladio \& M Lozada. 2006. Medicinal wild plant knowledge and gathering patterns in a Mapuche community of North-western Patagonia. J. Ethnopharmacol., 103:109-119.

EYSSARTIER, C; AH LAdio \& M LOZADA. 2009. Uso de plantas medicinales cultivadas en una comunidad semi-rural de la estepa patagónica. BLACMA, 8:77-85.

Eyssartier, C; AH Ladio \& M LozADA. 2011a.Traditional horticultural knowledge change in a rural population of the Patagonian steppe. J. Arid Environ., 75:78-86.

Eyssartier, C; AH Ladio \& M Lozada. 2011b. Horticultural and gathering practices complement each other: a case 
study in a rural population of Northwestern Patagonia. Ecol. Food Nutr., 50:429-451.

Ezcurra, C \& C Brion. 2005. Plantas del Nahuel Huapi. Catálogo de la Flora Vascular del Parque Nacional Nahuel Huapi, Argentina. Universidad Nacional del Comahue.

Golluscio, RA; ME Román; A Cesa; D Rodano; H Bottaro; ET AL. 2010. Aboriginal settlementes of arid Patagonia: Preserving-or sociodiversity? The case of the Mapuche pastoral Cushamen Reserve. J. Arid Environ., 74:13291339.

GonzÁlez-Insuasti, MS \& J Caballero. 2007. Managing plant resources: How intensive can it be? Human Ecol., 35:303-314.

Gundermann, H; J Canihuan; A Clavería \& C Faúndez. 2009. Permanencia y desplazamiento, hipótesis acerca de la vitalidad del mapuzugun. Revista de Linguística Teórica y Aplicada, 47:37-60.

GutiérRez, G. 2006. La Minería del Oro. Explotación a cielo abierto con utilización de cianuro: balance de beneficios y riesgos. Departamento de Pastoral Social. Ediciones ICEPH. Bariloche.

HÖFT, M; SK BARIK \& AM LYKKE. 1999. Quantitative Ethnobotany. Applications of multivariate and statistical analyses in ethnobotany. Ed. People and Plant Working Paper 6. UNESCO, Paris.

INDEC. 2001. Censo Nacional de Población, Hogares y Viviendas 2001 . Instituto Nacional de Estadísticas y Censos, Ministerio de Economía, Buenos Aires. Argentina.

ISE. 2006. International Society of Ethnobiology Code of Ethics._ethnobiology.net/code-of-ethics; último acceso: 10-12-2011.

Izquierdo, F; V Velasco \& A Nasif. 2009. Montes leñeros y cortinas de reparo en la Región Sur de Río Negro. INTA, Bariloche. Argentina.

LADIO, AH. 2011a. Underexploited wild plant foods of North- Western Patagonia. Pp. 1-16 en: Filipi, R (ed.). Multidisciplinary Approaches on Food Science and Nutrition for the XXI Century. Transworld Research Network, Kerala.

LADIO, AH. 2011b. Traditional knowledge of edible wild native and exotic plants in the context of cultural change in human populations of arid Patagonia. Biorem. Biodiv. Bioavail., 5:81-84.

Ladio, AH \& M Lozada. 2003. Comparison of wild edible plant diversity and foraging strategies in two aboriginal communities of northwestern Patagonia. Biodiv. Conserv., 12:937-951.

Ladio, AH \& M Lozada. 2004a. Patterns of use and knowledge of wild edible plants in distinct ecological environments: A case study of a Mapuche community from Northwestern Patagonia. Biodiv. Conserv., 13: 1153-1173.

Ladio, AH \& M Lozada. 2004b. Summer cattle transhumance and wild edible plant gathering in a Mapuche community of NW Patagonia. Hum. Ecol., 32:225-240.

LADIO, AH \& M LOZADA. 2008. Medicinal plant knowledge in rural communities of North-western Patagonia, Argentina. A resilient practice beyond acculturation. Pp. 40-53 en: Albuquerque, UP \& MA Alves Ramos (eds.). Current Topics in Ethnobotany. NUPEEA, Brazil.

Ladio, AH \& S Molares. 2010. Aspectos do estudo da dinâmica do uso de produtos etnobiológicos não tradicionais. Pp. 367-378, Vol. 1, $1^{\text {a }}$ ed. en: Albuquerque, UP; RF Paiva de Lucena \& LVFC Cunha (eds.). Métodos e Técnicas na Pesquisa Etnobiológica e Etnoecológica. NUPEEA, Recife, Brazil.

Lucena, RFP; UP Albuquerque; JM Monteiro; CDFCBR AlmeIDA \& ATN FlorentinO. 2007. Useful plants of the Semi-Arid Northeastern Region of Brazil. A look at their conservation and sustainable use. Environ. Monit. Assess., 125:281-290.

Molares, S \& AH LAdio. 2008. Plantas medicinales en una comunidad Mapuche del NO de la Patagonia Argentina: clasificación y percepciones organolépticas relacionadas con su valoración. BLACPMA., 7:149-155.

Molares, S \& AH Ladio. 2010. Métodos micrográficos aplicados à pesquisa etnobotânica. Pp. 381-399 en: Albuquerque, UP; RFP Lucena \& LVFC Cunha (eds.). Métodos e Técnicas na Pesquisa Etnobiológica e Etnoecológica. NUPEEA. Brasil.

Paruelo, JM; FJ Guerschman; G Piñeiro; EG Jobbágy; SR Verón; ET AL. 2006. Cambios en el uso de la tierra en Argentina y Uruguay: marcos conceptuales para su análisis. Agrociencia, 10:47-61.

Richeri, M; AH LADIO \& AM BEEsKow 2010. Etnoecología en la Patagonia árida: la adaptación de la herbolaria de una comunidad inmigrante boliviana a un nuevo contexto ambiental. Pp. 203-227 em: Chaves Alves, Bezerra Souto e Peroni (eds.). Etnoecologia em perspectiva: natureza, cultura e conservação. NUPEEA, Brasil.

Richeri, M; AH LAdIO \& AM BEesKow. 2012. Conocimiento tradicional y autosuficiencia: la herbolaria rural en la Meseta Central del Chubut (Argentina). BLACPMA, 12:48-58.

Romo, M; V Castro; C Villagrán \& C Latorre. 1999. La transición entre las tradiciones de los oasis del desierto y de las quebradas altas de Loa superior: Etnobotánica del valle del Río Grande, $2^{\mathrm{a}}$ Región, Chile. Chungara, Revista de Antropología Chilena, 31:319-360.

Santos Fita, D; EM Costa Neto \& EJ Cano-Contreras. 2009. El quehacer de la etnozoología. En: Costa Neto, ED; D Santos Fitas \& M Vargas Clavijo (eds.). Manual de Etnozoología. Tundra ediciones. Valencia.

Silva, VA; LHC Andrade \& UP Albuquerque. 2006. Revising the cultural significance index: the case of the Fulni-ô in northeastern Brazil. Field Methods, 18:98-108.

Soares Ferreira JRW; AH Ladio \& UP Albuquerque. 2011. Resilience and adaptation in the use of medicinal plants with suspected anti-inflammatory activity in the Brazilian Northeast. J. Ethnopharm., 138:238-252.

SUÁREZ, ME. 2009. El análisis de narrativas en etnobotánica: el "yuchán" (Ceiba chodatii, bombacaceae) en el discurso de los wichís del chaco semiárido salteño, argentina. Bol. Soc. Argent. Bot., 44:405-419.

Toledo, VM. 2007. Metabolismos rurales: hacia una teoría económica y ecológica de la apropiación de la naturaleza. Rev. Iberoam. Econ. Ecol., 7:1-2.

Zuloaga, F \& O Morrone. 1999a. Catálogo de las plantas vasculares de la República Argentina I. Acanthaceae, Euphorbiaceae (Dicotyledoneae). Ed. Missouri Botanical Garden Press. Missouri, USA.

Zuloaga, F \& O Morrone. 1999b. Catálogo de las plantas vasculares de la República Argentina II. Fabaceae. Zygophyllaceae (Dicotyledoneae). Ed. Missouri Botanical Garden Press. Missouri, USA. 